\title{
IRDE and RDE electrochemical cells evaluation: comparison of electron and mass transfer
}

\author{
H. Van Parys ${ }^{1}$, E. Tourwé ${ }^{1}$, M. Depauw ${ }^{1}$, T. Breugelmans ${ }^{1,2}$, \\ J. Deconinck ${ }^{3} \&$ A. Hubin ${ }^{1}$ \\ ${ }^{1}$ Vrije Universiteit Brussel, \\ Department of Metallurgy, Electrochemistry and Materials Science, \\ Brussels, Belgium \\ ${ }^{2}$ Hogeschool Antwerpen, \\ Department of Industrial Sciences and Technology-Chemistry, Antwerp, \\ Belgium \\ ${ }^{3}$ Vrije Universiteit Brussel, Computational Electrochemistry Group, \\ Department of Electrical Engineering and Power Electronics, Brussels, \\ Belgium
}

\begin{abstract}
An inverted rotating disk electrode reactor (IRDE) is constructed to facilitate the study of electrochemically formed gas bubbles. The hydrodynamic and mass transfer characteristics of this new cell design are validated by means of an electrochemical reaction with known characteristics, i.e. the ferri/ferro cyanide redox reaction. This validation is done both qualitatively and quantitatively. The qualitative validation consists of comparing the entire polarization curve obtained in the IRDE with the one obtained in the classical RDE set-up under the same experimental conditions. To quantitatively validate the cell another approach was pursued. A previously developed statistically founded method to model an electrochemical system and to quantitatively determine its mass and charge transfer parameters is used. It is found that the mass and charge characteristics in both configurations agree very well.

Keywords: inverted rotating disk electrode, IRDE, ferri/ferro cyanide, modeling.
\end{abstract}




\section{Introduction}

The study of electrochemically formed gas bubbles on an electrode surface and their influence on mass transfer are of great industrial importance. The formation of these gas bubbles can have several effects on the electrode behaviour, which have to be taken into consideration when dealing with gas evolution reactions. For example if gas evolution takes place on the electrode surface, not only will it consume part of the current, it will also have an important effect on the local process parameters and current distribution. Although indispensable for reactor design and optimization of new and existing industrial processes, no general model has yet been developed to describe the influence of gas bubbles on mass transport. The rotating disk electrode configuration is most often used in deterministic studies of the reaction kinetics. Due to the controlled convection obtained by rotating the disk electrode, it is possible to distinguish between mass and charge transport control in surface reactions. But the RDE has one drawback when dealing with gas evolution reactions, namely the formed gas bubbles tend to stick to the electrode disk and shield the active electrode surface in this way. The inverted RDE configuration can provide a solution to this problem, since the electrode surface is facing upwards instead of downwards. This way the generated gas bubbles can detach either by buoyancy or be swept away by the rotational movement of the electrode. So the gas bubbles can rise freely to the surface and are no longer shielding the electrode. Although the change in position of the working electrode in the inverted RDE from the top to the bottom of the electrochemical cell seems to be a minor change in the experimental setup, it has to be validated whether the mass and charge transfer equations governing the classical $\mathrm{RDE}$ can be transferred to the inverted $\mathrm{RDE}$ configuration. Therefore, before going to electrochemical reactions involving gas evolution, a system needs to be chosen which has well-known mass transfer characteristics and where no influence of the gas bubbles on the flow exists. Since the ferri-/ferro cyanide redox system is extensively studied [1-5], it is chosen as a model system to validate the inverted rotating disk electrode configuration (IRDE). In literature [6-9] the mass transfer characteristics of the IRDE are tested by measuring limiting currents in the ferricyanide reduction system. Subsequently the diffusion coefficient is calculated from the KouteckyLevich plot and is compared to the ones available in literature. Since mass transfer also plays its role in the part of the curve where charge transfer is dominant, a more rigorous validation technique consists in considering the whole polarization curve. Moreover, the validation will not only be done in a qualitative way by comparing the polarization curves obtained in the classical rotating disk set-up versus the ones measured in the inverted one, but also in a quantitative way. A new statistically founded method to model an electrochemical system and to quantitatively determine its mass and charge transfer parameters is recently developed and will be used in this work [10]. We believe this is, compared to the technique of qualitatively comparing part of the experimental data, a much more powerful tool to validate an electrochemical cell. 
In this work an inverted rotating disk electrode is constructed and is validated in both a qualitative and a quantitative way. The aim is to determine the charge and mass transfer parameters of the ferri/ferro redox system obtained in both the classical and the inverted RDE set-up and to compare them. In this way it can be decided on a reliable and statistically founded basis whether the inverted RDE obeys the same hydrodynamic and mass transport conditions as the classical RDE set-up, providing a suitable instrument for the study of electrochemical reactions taking place with simultaneous gas evolution.

\section{Experimental}

Electrochemical measurements are performed with a three electrode configuration using a PGSTAT $100^{\circledR}$ potentiostat from Ecochemie ${ }^{\circledR}$, controlled by the GPES 4.8 software. The counter electrode is a large platinum grid and the reference electrode is a saturated $\mathrm{Ag} / \mathrm{AgCl}$ electrode (Schott-Geräte). The working electrode is a rotating disk electrode of platinum, made by embedding a platinum rod (Alfa Aesar, 99.99\% purity) in an insulating PVDF cylinder. The rotation speed of the working electrode is kept constant at 1000rpm. The polarization curves are obtained at a rate of $1 \mathrm{mVs}^{-1}$ to reach quasi steady-state measurements at each point of the curve. The step potential is fixed at $0.00015 \mathrm{~V}$ in order to measure the maximal amount of data points the software allows.

For the preparation of the solutions once-distilled and demineralized water and analytical reagents (all Merck p.a.) are used. Equimolar solutions of potassium ferro- and ferricyanide $0.005 \mathrm{M} \mathrm{K}_{3} \mathrm{Fe}(\mathrm{CN})_{6}$ en $\mathrm{K}_{4} \mathrm{Fe}(\mathrm{CN})_{6}$ are used. A $1 \mathrm{M} \mathrm{KCl}$ solution is used as supporting electrolyte. In this way the migration flux can be neglected and the activity constants and diffusion coefficients of the electro-active components $\mathrm{Fe}^{2+}$ and $\mathrm{Fe}^{3+}$ can be considered to remain constant during the oxidation/reduction process. Prior to each measurement the electrolyte is bubbled with nitrogen gas for 5 minutes in order to deoxygenate the electrolyte. In this way the oxygen reduction is reduced and doesn't interfere with the reduction of ferricyanide resulting in a flattened reduction plateau. The electrochemical cell is shielded from light in order to avoid the following photochemical decomposition of ferrocyanide [9], i.e.

$$
\begin{aligned}
& \mathrm{Fe}(\mathrm{CN})_{6}^{4-} \stackrel{\text { light }}{\longleftrightarrow} \mathrm{Fe}(\mathrm{CN})_{5}^{3-}+\mathrm{CN}^{-} \\
& \mathrm{CN}^{-}+\mathrm{H}_{2} \mathrm{O} \leftrightarrow \mathrm{HCN}+\mathrm{OH}^{-}
\end{aligned}
$$

All measurements are thermostatically controlled at $25 \pm 0.1^{\circ} \mathrm{C}$ by means of a waterjacket around the cell, of which the temperature is controlled by a thermostat (Lauda RE304).

To improve the reproducibility of the measurements the following standardized pretreatment of the electrode surface is applied [9]:

- Mechanical polishing of the platinum electrode on a rotating disk (Struers DP10, on cloth), successively using a diamond paste of $7 \mu \mathrm{m}$ and $1 \mu \mathrm{m}$ (Struers) 
- Ultransonic rinsing with deionized water followed by degreasing with chloroform, both in an ultrasonic bath $\left(\mathrm{Elma}^{\circledR}\right.$ model T470/H) for 3 minutes

- Before each experiment, the electrode potential was swept 3 times between $+0.55 \mathrm{~V}$ and $-0.45 \mathrm{~V}$ vs. $\mathrm{Ag} / \mathrm{AgCl}$ sat., at a scan velocity of $0.01 \mathrm{~V} / \mathrm{s}$ and a rotation speed of $1000 \mathrm{rpm}$. It is reported in literature [11], that such a pretreatment removes oxide and trace contaminants from the Pt surface, while the $\mathrm{O}_{2}$ and $\mathrm{H}_{2}$ evolution reactions are avoided in this potential range.

Before each experiment this electrode pretreatment procedure is repeated.

\section{Results and discussion}

\subsection{Cell design of the IRDE}

As in the classical RDE set-up, the inverted configuration consists of a cylindrical vessel with a rotating working electrode, now placed at the bottom of the cell, a counter electrode positioned at a fixed point at the top of the vessel and the reference electrode also placed at the top of the cylindrical cell (see Figure 1). A construction of O-rings keeps the electrolyte from leaking at the bottom of the rotating electrode. The main difference between the two configurations is that in the inverted RDE set-up the working electrode is protruding into the solution what can lead to the creation of additional vortices in the flow introducing a different flow pattern compared to the classical RDE.

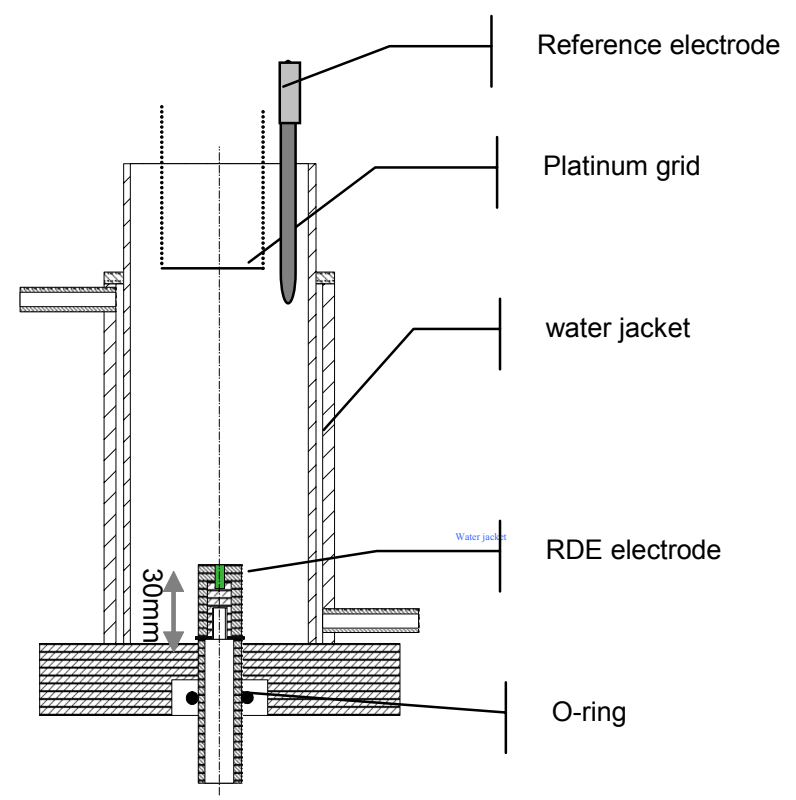

Figure 1: $\quad$ Sketch of the IRDE cell (front view), not a scale drawing. 


\subsection{Validation of the inverted RDE configuration}

The reduction of ferricyanide to ferrocyanide at a platinum electrode is an example of a relatively simple electrode process in which an electron is exchanged across the electrode/electrolyte interface and in which no complex mechanism is expected to take place, i.e.

$$
\mathrm{Fe}(\mathrm{CN})_{6}^{3-}+e^{-} \leftrightarrow \mathrm{Fe}(\mathrm{CN})_{6}^{4-}
$$

As advised in [10] at least 11 polarization curves with the same experimental conditions are recorded in the inverted RDE. Exactly the same experiments are performed in the RDE set-up in the papers of E. Tourwe $[12,13]$. The latter experimental series are considered to be reference data to compare our data to.

\subsubsection{Qualitative validation of the mass transfer characteristics in IRDE}

The mean polarization curve is calculated from the 11 polarization curves by calculating the mean of the current at each data point. The $95 \%$ confidence interval is obtained in a similar way, namely by calculating the standard deviation of the mean current at each data point. The $95 \%$ confidence interval is then given by $+/-2$ times the standard deviation. This interval gives the range of current values that is expected to include $95 \%$ of all experimental data. The mean polarization curves and their respective confidence intervals obtained in both the classical and the inverted RDE configuration are compared in Figure 2. Note that the confidence interval for the IRDE is very small and therefore difficult to observe in figure 2 . In the inset, a magnification of an arbitrary part of the polarization curve is shown. It is seen that the $95 \%$ confidence interval obtained in the inverted RDE falls within the one obtained in the classical RDE. So on an experimental basis it can be concluded that the same polarization curves are measured in both configurations and that they compare well. Remark that the confidence interval of the polarization curve obtained in the IRDE is smaller that the one obtained in the RDE. This could indicate that the IRDE is a more accurate tool to measure reaction kinetics than the RDE. Yet, this still has to be established by an extended set of data under different experimental conditions, such as rotating speed, temperature, etc.

\subsubsection{Quantitative validation of the mass transfer characteristics in IRDE}

To determine the kinetic behavior of the system under study, the methodology described in the papers written by E. Tourwé $[10,12]$ is used. Therefore an appropriate reaction mechanism has to be proposed. This mechanism is then translated in a mathematical expression that analytically describes the polarization curve. In a next step powerful parameter estimation algorithms (maximum likelihood combined with Gauss-Newton and Levenberg Marquardt minimization methods) are used to adjust the values of these model parameters, in order to obtain a good agreement between experimental and modeled data. When a good match between the experimental and simulated data is achieved, 
the model can be retained and the kinetic parameters of the system are quantified. However it is only after a statistical evaluation of the fit that it is decided whether the model describes the system well.

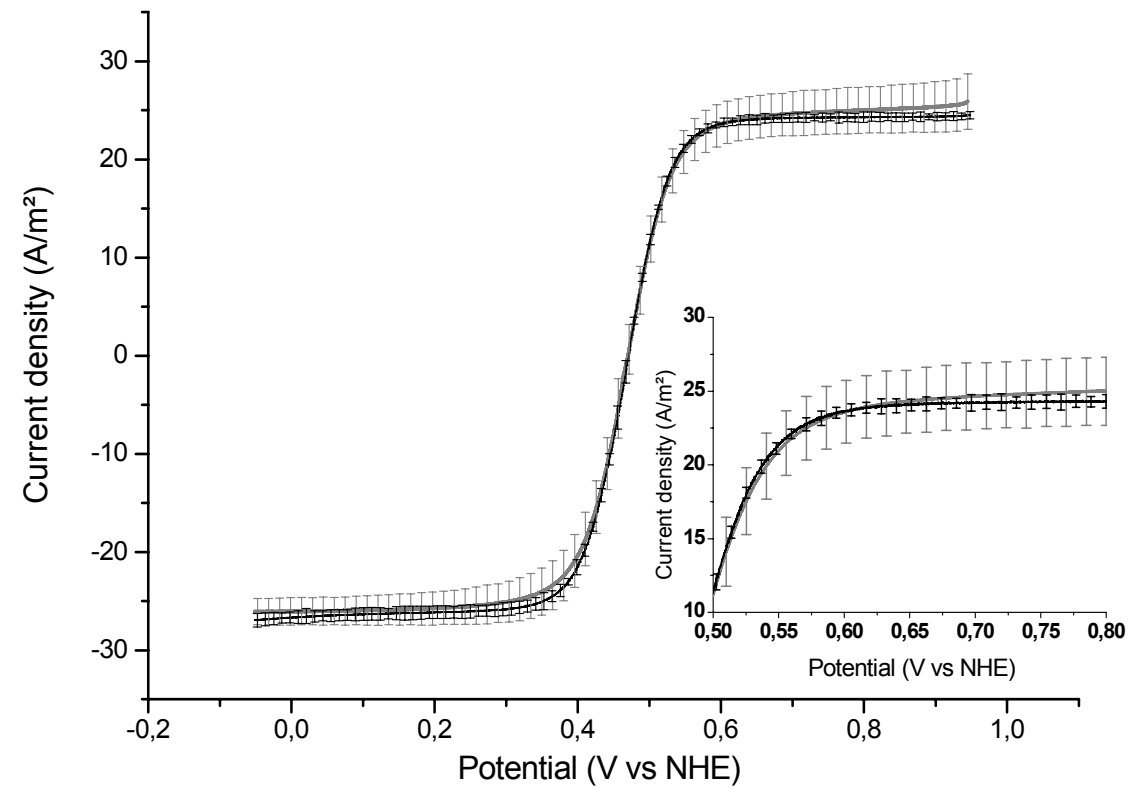

Figure 2: Mean voltammogram of the reduction/oxidation of $0,005 \mathrm{M}$ ferri/ferrocyanide in $1 \mathrm{M} \mathrm{KCl}$ obtained in the classic RDE set-up (grey) and in the inverted RDE set-up (black) and their respective $95 \%$ confidence interval, at $1000 \mathrm{rpm}$.

The mathematical expression believed to describe the current densitypotential relationship of the electrochemical reaction (see eqn (2)) is the following set of equations. These expressions take into account mass and charge transfer.

$$
\begin{gathered}
r e d \leftrightarrow o x+n e^{-} \\
j=\frac{n F\left(K_{o x} c_{r e d}^{*}-K_{r e d} c_{o x}^{*}\right)}{\frac{1}{S}+n F\left(\frac{K_{o x} c_{r e d}^{*}}{i_{\text {lim }, o x}}-\frac{K_{r e d} c_{o x}^{*}}{i_{\text {lim }, r e d}}\right)} \\
\text { with: } K_{o x}=k_{o x} \exp \frac{\alpha_{o x} n F E}{R T} \\
K_{r e d}=k_{r e d} \exp \frac{-\alpha_{r e d} n F E}{R T}
\end{gathered}
$$


$\mathrm{j}: \quad$ the current density $\left(\mathrm{A} / \mathrm{m}^{2}\right)$

$\mathrm{n}$ : the number of electrons exchanged, here 1

F: $\quad$ Faraday's constant $(96485 \mathrm{C} / \mathrm{mol})$

S: $\quad$ electrode surface $\left(\mathrm{m}^{2}\right)$

$\mathrm{K}_{\mathrm{ox}}$ : the potential dependent rate constant for the oxidation half reaction

$\mathrm{K}_{\text {red }}$ : the potential dependent rate constant for the reduction half reaction

$\mathrm{E}: \quad$ the potential (V vs. NHE)

$\mathrm{R}: \quad$ ideal gas constant $\left(8,32 \mathrm{~J} \cdot \mathrm{mol}^{-1} \cdot \mathrm{K}^{-1}\right)$

$\mathrm{T}$ : the absolute temperature $(\mathrm{K})$

$c_{\text {red }}^{*}$ : the bulk concentration of the reducing agent $\left(\mathrm{mol} / \mathrm{m}^{3}\right)$, in this case $\mathrm{Fe}(\mathrm{CN})_{6}^{4-}$

$c_{o x}^{*}$ : the bulk concentration of the oxidizing agent $\left(\mathrm{mol} / \mathrm{m}^{3}\right)$, in this case $\mathrm{Fe}(\mathrm{CN})_{6}^{3-}$

$\mathrm{i}_{\text {lim, ox }}$ : the oxidation limiting current $(\mathrm{A})$

$\mathrm{i}_{\text {lim, red }}$ : the reduction limiting current $(\mathrm{A})$

$\mathrm{k}_{\mathrm{ox}}$ : the rate constant for the oxidation half reaction $(\mathrm{m} / \mathrm{s})$

$\mathrm{k}_{\mathrm{red}}$ : the rate constant for the reduction half reaction $(\mathrm{m} / \mathrm{s})$

$\alpha_{\mathrm{ox}}$ : the transfer coefficient for the oxidation

$\alpha_{\text {red }}$ : the transfer coefficient for the reduction

The theoretical expression will be fitted to the mean polarization curve obtained in the inverted RDE configuration (see Figure 2), using the fitting algorithm. In Figure 3 the fitting results are represented. Part a shows the fitted and the experimental polarization curve and the $95 \%$ confidence interval of the experiments. As can be seen, the fitted plot concurs very well with the experimental polarization curve. In part $b$ of Figure 3 the residual plot is shown in which the difference between the calculated and the experimental data is represented as a function of the potential. This graph gives a clear visual representation of the goodness-of-fit. If the residuals fall within the $95 \%$ confidence interval, a good fit is obtained. The graph shows that the residuals indeed fall within the $95 \%$ confidence interval. So we consider this to be a good match and the model put forward in eqn (2) is able to describe the kinetics of the ferri/ferro redox reaction in the IRDE.

In table 1 the quantified model parameters are represented and compared to these obtained in the classical RDE $[12,13]$. The diffusion coefficients are not calculated here because in this paper only one rotation speed is considered. In order to calculate the diffusion coefficients in a good way, a data set taken at different rotation speeds is needed. Table 1 shows that the parameters agree well. We have to point out that the $95 \%$ confidence interval for the respective parameters obtained in the RDE and IRDE configuration don't overlap, although they lie very close to one another. This doesn't necessarily mean that the values are inconsistent. The values are so close that it shows that these parameters are very sensitive to the starting condition of the working electrode which cannot be fully controlled e.g. the presence of dislocations, scratches, etc. Moreover the parameters agree very well with the values found in literature. Literature values 
for $\alpha_{\mathrm{ox}}$ vary between 0.45 and $0.61[3,4]$. When the rate constants of table 1 are calculated (see eqn (6)) taking into account the equilibrium constant $\mathrm{E}_{0}=0.47 \mathrm{~V} / \mathrm{NHE}$ the following values are obtained: $\mathrm{k}_{\mathrm{ox}}^{\prime}=1.98 \mathrm{E}-04 \mathrm{~m} / \mathrm{s}^{\prime}$ and $\mathrm{k}_{\text {red }}$ $=1.94 \mathrm{E}-04 \mathrm{~m} / \mathrm{s}$.
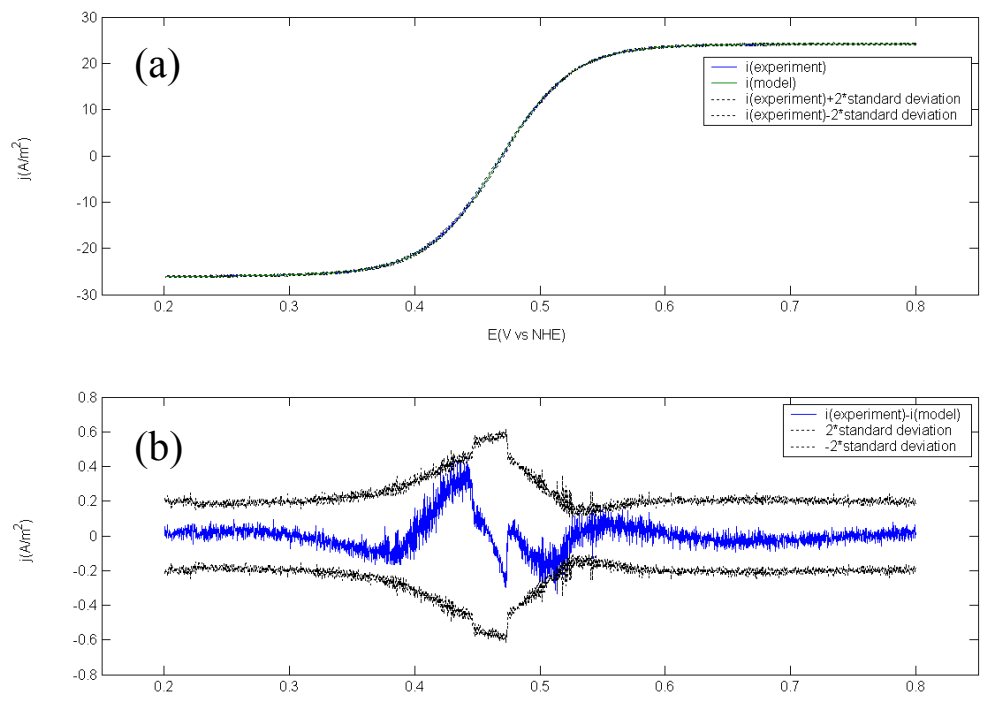

Figure 3: Comparison of the mean experiment obtained in the IRDE with the model, at 1000rpm: (a) the calculated and the simulated polarization curve together with the $95 \%$ confidence interval, (b) the difference between the experimental and simulated polarization curve at each potential together with the $95 \%$ confidence interval.

Table 1: Kinetic parameters of the ferri/ferrocyanide redox reaction with the respective absolute standard deviation.

\begin{tabular}{|l|c|c|}
\hline & Classical RDE & Inverted RDE \\
\hline $\boldsymbol{\alpha}_{\boldsymbol{o x}}$ & $\mathbf{5 . 0 0 E - 1} \pm 8.76 E-03$ & $\mathbf{5 , 3 7 E - 0 1} \pm 3.87 e-03$ \\
\hline $\boldsymbol{k}_{\text {ox }}$ & $\mathbf{1 . 3 9 E - 0 8} \pm 2.28 E-09$ & $\mathbf{1 , 0 6 E - 0 8} \pm 7.70 E-10$ \\
\hline $\boldsymbol{k}_{\boldsymbol{r e d}}$ & $\mathbf{1 . 2 5 E + 0 0} \pm 2.01 E-01$ & $\mathbf{9 , 3 3 E - 0 1} \pm 6.66 E-02$ \\
\hline $\boldsymbol{j}_{\text {lim, }, \boldsymbol{o x}}$ & $\mathbf{2 . 4 7 E + 0 1} \pm 1.57 E-02$ & $\mathbf{2 , 4 2 E + 0 1} \pm 7.65 E-03$ \\
\hline $\boldsymbol{j}_{\text {lim, }, \boldsymbol{r e d}}$ & $\mathbf{- 2 . 5 6 E + 0 1} \pm 1.43 E-02$ & $-\mathbf{2 , 6 1 E}+\mathbf{0 1} \pm 1.14 E-02$ \\
\hline
\end{tabular}

$$
k_{o x / \text { red }}^{\prime}=k_{o x / \text { red }} \exp \frac{\alpha_{o x} n F E_{0}}{R T}
$$

These are of the same order of magnitude as the ones reported in literature, which range from $5 \mathrm{E}-04 \mathrm{~m} / \mathrm{s}$ until $1 \mathrm{E}-03 \mathrm{~m} / \mathrm{s}$ [3-5,14-15]. 


\section{Conclusions}

It is shown that the IRDE obeys the same hydrodynamic conditions as the classical RDE set-up. It has the same advantages of well-defined mass transport conditions. In future work, the setup will be used for the study of the kinetics of gas evolution reactions taking advantage of the fact that the formed gas bubbles can freely rise to the surface.

Secondly it is shown that the fitting algorithm is a very powerful and sensible tool to validate the mass transfer characteristics of a new reactor, such as the IRDE. The validation was done making use of the ferri/ferro cyanide reference system. The values of the charge transfer parameters are: $\alpha_{o x}=5,37 \mathrm{E}-01 \pm 3.87 \mathrm{e}-$ $03, \mathrm{k}_{\mathrm{ox}}=1,06 \mathrm{E}-08 \pm 7.70 \mathrm{E}-10 \mathrm{~m} / \mathrm{s}$ and $\mathrm{k}_{\mathrm{red}}=9,33 \mathrm{E}-01 \pm 6.66 \mathrm{E}-02 \mathrm{~m} / \mathrm{s}$.

\section{Acknowledgements}

This research is funded by the Flemish Institute for support of ScientificTechnological Research in Industry (IWT SBO contract number 040092, project acronym: Mutech and post-doc position of E. Tourwé).

\section{References}

[1] Bamford, C. \& Compton R., Electrode Kinetics: Principles and Methodology, volume 26 of Comprehensive Chemical Kinetics, Elsevier Science Publishers, 1986

[2] Iwasita, T., Schmickelr, W., Hermann, J. \& Vogel, U. Journal of Electrochemical Society, 130, p. 2026, 1983

[3] Angell, D. \& Dickinson, T. Journal of Electroanalytical Chemistry, 35, p. 55,1972

[4] Bruce, P., Lisowska-Oleksiak, A., Los, P. \& Vincent, C. Journal of Electroanalytical Chemistry, 367, p.279, 1994

[5] Beriet, C. \& Pletcher, D. Journal of Electroanalytical Chemistry, 361, p.93, 1993

[6] Zdunek, A.D. \& Selman, J.R. Journal of the Electrochemical Society, 139 (9), p.2549, 1992

[7] Bressers, P.M.M.C. \& Kelly, J.J. Journal of the Electrochemical Society, 142 (7), p. L114, 1995

[8] Bradley, P.E. \& Landolt, D. Journal of the Electrochemical Society, 144 (6), p. L145, 1997

[9] Vandeputte S., PhD Thesis, Vrije Universiteit Brussel, Brussels, 1996

[10] Tourwé, E., Breugelmans, T., Pintelon, R. \& Hubin, A. Journal of Electroanalytical Chemistry, 594(1), p. 50, 2006

[11] Robertson, B., Tribollet, B. \& Deslouis, C. Journal of Electrochemical Society, 135, p. 2279, 1988

[12] Tourwé, E., Pintelon, R. \& Hubin, A. Journal of Electroanalytical Chemistry, accepted

[13] Tourwé, E., Pintelon, R. \& Hubin, A. submitted to this journal 
192 Simulation of Electrochemical Processes II

[14] Jahn, D., Vielstich, W. Journal of Electrochemical Society, 109, p.849, 1962

[15] Tanaka, N., Tamamushi, R. Electrochimica Acta, 9,p. 963, 1964 